\title{
Métodos diagnósticos en candidemia: una revisión sistemática de la literatura con meta-análisis
}

\author{
Jorge Alberto Cortés, Alejandro Concha M., Luis Eduardo Cediel T. y Juan Sebastián Castillo
}

\section{Diagnostic methods in candidemia: a systematic review of literature with meta-analysis}

Candidemia is a disease with high morbidity and mortality especially in critical care patients. Early diagnosis enables early treatment. Objectives: To conduct a systematic review of the literature in order to establish the best laboratory tests for the diagnosis of candidemia in critical patients. Materials and Methods: We conducted a systematic review of available literature in PubMed. Serological studies were subjected to meta-analysis in metadisk-Beta 1.1.1. Results: 4 studies of 1286 reviewed were included. Three were about serological tests and one about molecular testing (RT-PCR). The sensitivity and specificity for RT-PCR, antibody testing and antigen and antibody tests were $87 \%$ and $100 \%, 47.5 \%$ and $82.6 \%, 96 \%$ and $81 \%$, respectively. Diagnostic Odds Ratio of antigenemia was $1.51(95 \% \mathrm{CI}=0,032-70,964, \mathrm{p}=0.001)$. Conclusions: RT-PCR has better diagnostic performance, measuring antigenemia plus antibodies improves sensitivity, specificity, LR + and LR- ᄀ. There is insufficient evidence to support this.

Key words: Fungemia, candidemia, molecular diagnostic techniques, serology, systematic reviews.

Palabras clave: Fungemia, candidemia, técnicas de diagnóstico molecular, serología, revisión sistemática.

\section{Introducción}

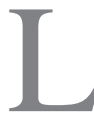
a candidiasis sistémica es una patología con alta morbilidad y mortalidad ${ }^{1,2}$. Ésta se presenta con una alta frecuencia en la población hospitalizada, especialmente aquella sometida a servicios de cuidado crítico $^{2}$. Esto genera no sólo un elevado impacto en los indicadores de morbilidad y mortalidad en los servicios de atención sanitaria, sino un impacto creciente en el costo asociado a esta entidad ${ }^{1}$.

Algunos factores como las largas hospitalizaciones en unidades de cuidados intensivos (UCI), la inmunosupresión subyacente en los pacientes (Ej. enfermedades auto-inmunes, quimioterapia, administración de corticoesteroides, malignidad hematológica o tumor sólido, neutropenia y trasplante de órganos), la terapia antimicrobiana, los factores mecánicos que interfieren las barreras naturales (Ej. quemaduras, uso de catéteres, implante de prótesis, ventilación mecánica), son factores asociados a la emergencia de esta patología ${ }^{2,3}$.

En Colombia, datos del grupo para el control de la resistencia bacteriana (GREBO) muestran que entre 2 y $5 \%$ de los aislados del torrente sanguíneo en UCI son ocasionadas por especies de Candida ${ }^{4}$. En general, se ha demostrado que Candida albicans es la especie asociada con mayor frecuencia a infecciones sistémicas del torrente sanguíneo, pero que su frecuencia en las UCI ha disminuido en el tiempo ${ }^{5}$. Dado el impacto clínico y la frecuencia de presentación de candidemias, especialmente en pacientes gravemente comprometidos, se hace necesario disponer de herramientas diagnósticas, que permitan una rápida y adecuada determinación de la candidemia y que faciliten la tipificación etiológica entre las especies de Candida, dada la diferencia registrada entre la mortalidad atribuible y factores de riesgo asociados para C. albicans y especies de Candida no albicans ${ }^{6}$.

El diagnóstico temprano permite realizar un tratamiento empírico adecuado en etapas tempranas de la enfermedad y esto genera una mayor posibilidad de afectar la alta mortalidad de esta patología ${ }^{7}$. Sin embargo, a pesar de las diferentes técnicas disponibles en la literatura científica, no se conocen con exactitud cuáles serían las de mejor rendimiento y su funcionamiento en nuestro medio, especialmente en pacientes en condiciones críticas.

\section{Materiales y Métodos}

Se realizó una revisión sistemática de la literatura médica, para identificar y resumir todas las publicaciones relacionadas con técnicas diagnósticas para candidemia en pacientes hospitalizados en UCI, así como sus características operativas, las poblaciones y condiciones de los sujetos evaluados. Se realizaron selecciones de artículos
Universidad Nacional de Colombia, Bogotá, Colombia. Departamento de Medicina Interna (JAC, ACM, LECT). Departamento de Epidemiología (JSC).

Realizado en la Universidad Nacional de Colombia, Facultad de Medicina, Departamento de Medicina Interna, sede Bogotá D.C.

Financiación: Los autores declaran que esta investigación se realizó con fondos provenientes de la Dirección de investigaciones Sede Bogotá de la Universidad Nacional de Colombia (código DIB 202010012688).

Recibido: 10 de diciembre de 2010 Aceptado: 4 de mayo de 2011

Correspondencia a: Jorge Alberto Cortés jorgecortes@yahoo.com 
relevantes guiados por título y resumen hasta obtener estudios clínicos originales. Se efectuó la búsqueda de información en la siguiente base de datos: MEDLINE (PubMed; enero de 1966 a diciembre de 2008). Las estructuras de búsqueda se diseñaron usando palabras texto identificadas en artículos relevantes, palabras clave según la base de datos consultada y sinónimos extractados de las bases de datos y publicaciones disponibles en idioma inglés. Se usaron filtros y estrategias validadas para la búsqueda de artículos relacionados con pruebas diagnósticas ${ }^{2}$. La estrategia de búsqueda término a término fue la siguiente (((() candid* OR candida OR fung*) AND (((candida[tw] OR candida) AND (blood* OR blood*[tw])) OR ((Fungemia[tw] OR Fungemias[tw] OR (bloodstream[tw] AND infect*[tw])) OR ((Sepsis[tw] OR Severe Sepsis[tw] OR Sepsis, Severe[tw] OR Septicemia[tw]

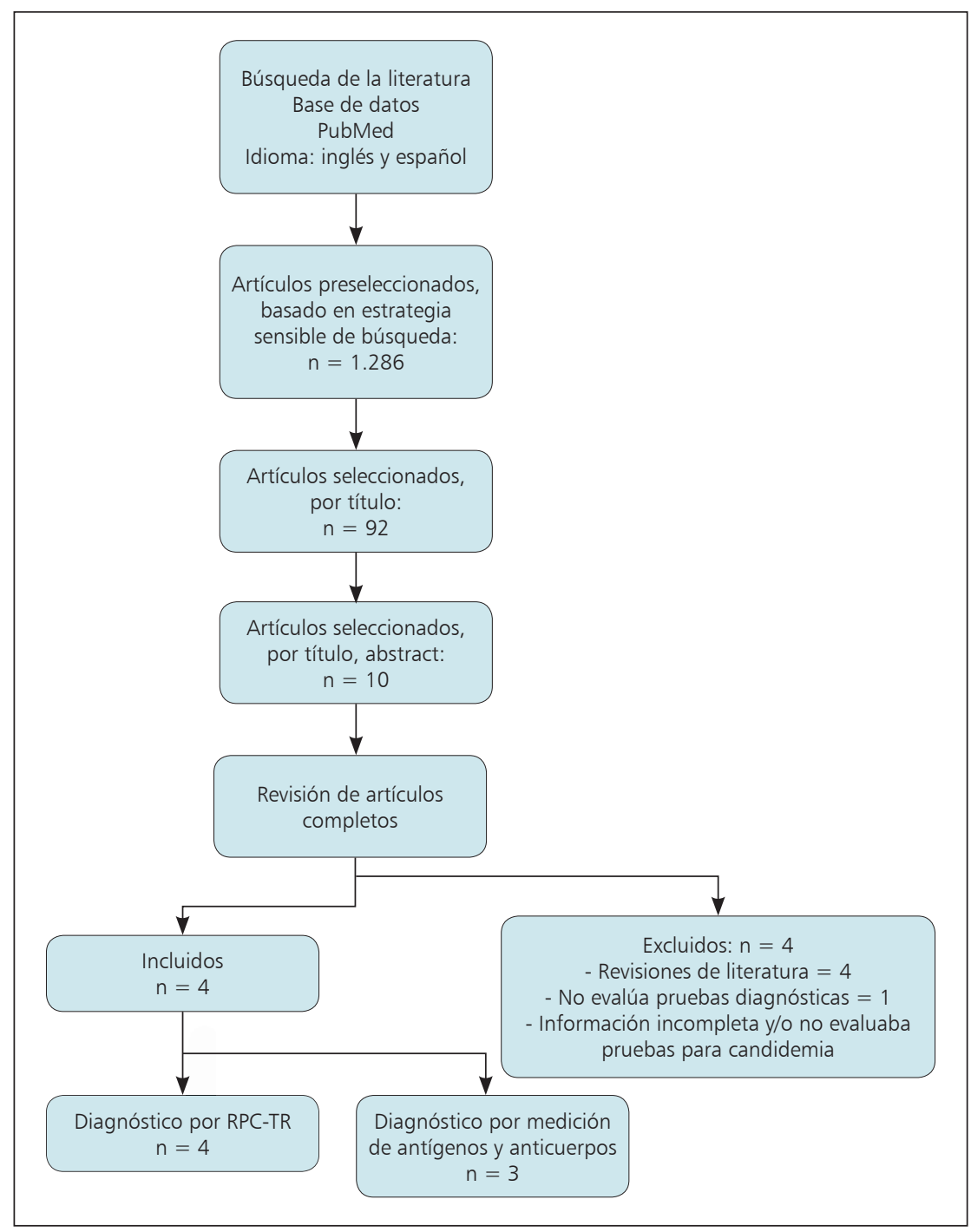

Figura 1. Selección de artículos.
OR Blood Poisoning[tw] OR Poisoning, Blood[tw]) AND (candida[tw])) OR (Invasive Candida infection[adj] OR Candidemia OR candidiases OR candidiasis OR invasive fungal infection[adj] OR Systemic candidiasis[adj] OR Systemic Candida[adj] invasive candidal infections[adj] OR Candidaemia) $)))$ ) AND ((*polymerase* OR DNA OR smear OR diagnostic* OR Antigen* OR Test* OR Immunoassay* OR Inmuno* OR Mannan* OR PCR* OR Molecular* OR Assay* OR germ* tube[adj] OR germ tube antibodies[adj] OR mannan OR Fluorescent OR fluorescence OR Immunolabeling* OR Immunogold* OR Immunohistocytochemistry OR glucan OR ELISA OR FISH OR CHROMagar OR peptide nucleic acids[adj] OR Antigen* OR kit*))). Se evaluó de manera pareada (ACM, LEC) la elegibilidad de los artículos a partir de los títulos de las referencias y resúmenes. Se extractó la información relacionada con las características operativas de las pruebas evaluadas, poblaciones de los estudios, escenarios de aplicación y limitaciones o amenazas a la validez de los mismos. Se consolidó la información en una base de datos en el programa MetaDisc-Beta. Los datos obtenidos para pruebas serológicas se unieron a través de un meta-análisis y se obtuvo la razón de probabilidades diagnóstica, sensibilidad, especificidad, coeficientes de probabilidades positiva y negativa. Se evaluó la heterogeneidad de los resultados por medio de $\chi^{2}$ y el sesgo de publicación con las herramientas estadísticas aceptadas y análisis de efecto umbral con coeficiente de correlación de spearman. Finalmente se generó un reporte de acuerdo a las recomendaciones PRISMA ${ }^{8}$.

\section{Resultados}

Sólo se identificaron cuatro estudios para el análisis final de esta revisión ${ }^{9-12}$. Tres de éstos evaluaban pruebas diagnósticas basadas en serología (antigenemia y anticuerpos) y uno utilizaba una prueba de diagnóstico molecular (RPC-TR ADN) (Figura 1). Ningún estudio presentó asignación aleatoria ni se efectuó cegamiento en las diferentes partes del estudio y todos tenían en los criterios de inclusión criterios clínicos. Al analizar la información en la etapa de lectura completa de texto, encontramos que se podía realizar meta-análisis de los que incluían pruebas serológicas; éstas se hicieron por pruebas de anticuerpos, pruebas de antígenos y sólo en dos se pudieron mezclar los datos de las pruebas antigénicas y de anticuerpo y realizar un meta-análisis de éstas (Tabla 1).

\section{Reacción de polimerasa en cadena en tiempo real (RPC-TR)}

En un estudio prospectivo observacional diagnóstico ${ }^{12}$, McMullan y cols., analizaron en dos etapas clínicas el rendimiento diagnóstico de una prueba de RPC-TR 
Tabla 1. Características de los estudios incluidos. Métodos diagnósticos para candidemia en unidades de cuidados intensivos (UCI)

\begin{tabular}{|c|c|c|c|c|c|c|}
\hline Estudio & Año & Tamaño (n) & Tipo de estudio & Prueba evaluada & Criterio de inclusión & Tiempo de duración \\
\hline Ibáñez-Nolla & 2001 & 93 & Cohorte prospectiva & $\begin{array}{l}\text { Anticuerpos } \\
\text { - Hasta enero de } 1994 \text { hemaglutinación } \\
\text { - Desde enero de 1994, inmunofluores- } \\
\text { cencia indirecta - tubo germinal } \\
\text { Antigenemia } \\
\text { - Hasta enero de } 1994 \text { medición de } \\
\text { antígenos termolábiles por Cand-Tec }{ }^{\circledR} \\
\text { - Desde enero de 1994, medición de } \\
\text { manano por Ac monoclonales por } \\
\text { Pastorex®. } \\
\text { Probabilidad clínica }\end{array}$ & $\begin{array}{l}\text { Pacientes en UCI con } \\
\text { al menos una muestra } \\
\text { orgánica positiva para } \\
\text { Candida sp }\end{array}$ & 1988 a 1995 \\
\hline Lepper y cols & 2001 & 24 & $\begin{array}{l}\text { Casos y controles, } \\
\text { retrospectivo }\end{array}$ & $\begin{array}{l}\text { Probabilidad clínica } \\
\text { Anticuerpos } \\
\text { - Hemaglutinación. Candida HAq®, } \\
\quad \text { Roche } \\
\text { Antigenemia } \\
\text { - CandTec } \circledast \text {, Ramco }\end{array}$ & Criterios clínicos & $\begin{array}{l}\text { Octubre de } 1996 \text { a } \\
\text { octubre de } 1999\end{array}$ \\
\hline Bär y cols & 2002 & $\begin{array}{c}104 \\
\text { (análisis hecho } \\
\text { sobre } 59 \text { ) }\end{array}$ & Prospectivo & $\begin{array}{l}\text { Anticuerpos } \\
\text { - Hemaglutinación indirecta } \\
\text { Antigenemia } \\
\text { - Cand-Tec } ® \text { latex }\end{array}$ & Criterios clínicos & SD \\
\hline Mc Mullan y cols & 2008 & 145 & $\begin{array}{l}\text { Prospectivo obser- } \\
\text { vacional diagnóstico }\end{array}$ & $\begin{array}{l}\text { Reacción de polimerasa en cadena en } \\
\text { tiempo real. TaqMan }\end{array}$ & $\begin{array}{l}\text { Pacientes con más de } 72 \\
\text { horas de hospitalización } \\
\text { en UCI } \\
\text { Criterios clínicos }\end{array}$ & SD \\
\hline
\end{tabular}

de ADN. En la primera recolectaron 515 muestras de 145 sujetos según los criterios de inclusión del estudio (EORTC), 491 muestras fueron negativas para RPCR-TR, y sólo tres de 13 muestras de un solo caso probable fueron positivas. De 11 especímenes de casos probados, nueve fueron positivos, sólo dos negativos; uno candidemia por C. albicans y otro por C. famata (esta última no hidridizó en la elaboración de partidores). En la segunda etapa sólo se incluyeron los que tenían definición de caso probado de candidemia, 12 especímenes; nueve por C. albicans, dos por C. glabrata y una mixta por estas dos. Once pruebas de RPC TR fueron positivas. Al hacer el análisis global de la prueba en cuanto a las muestras, la sensibilidad de la RPC TR ADN es de 87\% (20/23); el análisis hecho por los pacientes de los que se tomaron las muestras reporta sensibilidad de $87 \%$, especificidad de $100 \%$, valor predictor positivo $100 \%$, valor predictor negativo $99,6 \%$. Consideramos necesario mostrar el análisis si se incluyen los casos probables que finalmente llevan a tratamiento empírico en los cuales la sensibilidad cae a $64 \%$ por muestra y $72 \%$ por paciente.

\section{Pruebas serológicas}

Tres estudios incluidos en esta revisión sistemática evaluaban pruebas de serología para candidemia ${ }^{9-11}$.
Anticuerpos. Se encontró que las pruebas de anticuerpos fueron diferentes en cuanto a técnica y puntos de corte para positividad, problema que se ajustó por análisis del efecto umbral. La sensibilidad de las pruebas de detección de anticuerpos varió entre 36,1 y 87,5\%, la medición global fue de 47,5\% (IC 95\% = 34,6-60,7; p = 0,02), la especificidad fue de $82,6 \%$ (IC 95\% = 74,4-89) pero los datos no fueron estadísticamente significativos. Los demás indicadores de desempeño diagnóstico se encuentran en la Tabla 2.

La Odds Ratio diagnóstica (ORD) para candidemia en las pruebas de detección de anticuerpos fue de 6,085 (IC 95\% = 1,42-26,08; $\mathrm{p}=0$,047) ajustada por modelo de efectos aleatorios por el bajo tamaño de muestra, el estudio de Lepper y cols, con un peso del 19,9\% tiene la ORD individual más alta (Figura 2.)

Antigenemia. Los resultados para pruebas de medición antigenemia mostraron significancia estadística; sin embargo, baja sensibilidad 18\% (IC 95\% = 9,4-30), la especificidad fue alta con 90,4\% (IC 95\% = 83,5-95,1). La ORD para candidemia fue de 1,51 (IC 95\% = 0,03270,964; $\mathrm{p}=0,001$ ) (Figura 3). La prueba de $\chi^{2}$ demostró que sólo estos datos fueron homogéneos; no obstante, la poca cantidad de artículos limita el poder de esta apreciación. 
Tabla 2. Características operativas diagnósticas de las diferentes pruebas en los estudios incluidos

\begin{tabular}{|c|c|c|c|c|c|c|c|c|c|c|c|c|}
\hline \multirow[t]{2}{*}{ Estudio } & \multicolumn{4}{|c|}{ Anticuerpos } & \multicolumn{4}{|c|}{ Antigenemia } & \multicolumn{4}{|c|}{ Anticuerpos + antígenos } \\
\hline & $S(\%)$ & $E(\%)$ & $L R+$ & $L R-$ & $S(\%)$ & $E(\%)$ & $L R+$ & $L R-$ & $S(\%)$ & $E(\%)$ & $L R+$ & $L R-$ \\
\hline Ibáñez-Nolla & 36,1 & 78,9 & 1,715 & 0,809 & 0 & 89,5 & 0,121 & 1,111 & 87,5 & 75 & 3,5 & 0,167 \\
\hline Lepper y cols & 87,5 & 87,5 & 7 & 0,143 & 12,5 & 75 & 0,5 & 1,167 & 100 & 83,3 & 5,574 & 0,034 \\
\hline Bär y cols & 52,9 & 85,7 & 3,706 & 0,549 & 58,8 & 97,6 & 24,706 & 0,422 & SD & SD & SD & SD \\
\hline Global & 47,5 & 82,6 & 3,065 & 0,594 & 18 & 90,4 & 1,266 & 0,845 & 96 & 81 & 4,731 & 0,098 \\
\hline (IC95\%) & $34,6-60,7$ & $74,4-89$ & $1,41-6,62$ & $0,32-1,07$ & $9,4-30$ & $83,5-95,1$ & $0,05-29,42$ & $0,41-1,73$ & $79,6-99,9$ & $68,6-90,1$ & $2,79-8,01$ & $0,02-0,50$ \\
\hline Valor de $p$ & 0,02 & 0,579 & 0,117 & 0,054 & 0,000 & 0,034 & 0,003 & 0,000 & 0,124 & 0,479 & 0,408 & 0,292 \\
\hline
\end{tabular}

Antígenos más anticuerpos. Se encontraron dos estudios que tenían datos suficientes para la realización de análisis global uniendo las pruebas serológicas en conjunto; antígenos y anticuerpos; sensibilidad global de 96\% (IC 95\% = 79,6-99,9; p = 0,124). Con una ORD de 49,697 (IC 95\% = 6,43-383,94; $\mathrm{p}=0,27$ ) (Figura 4).

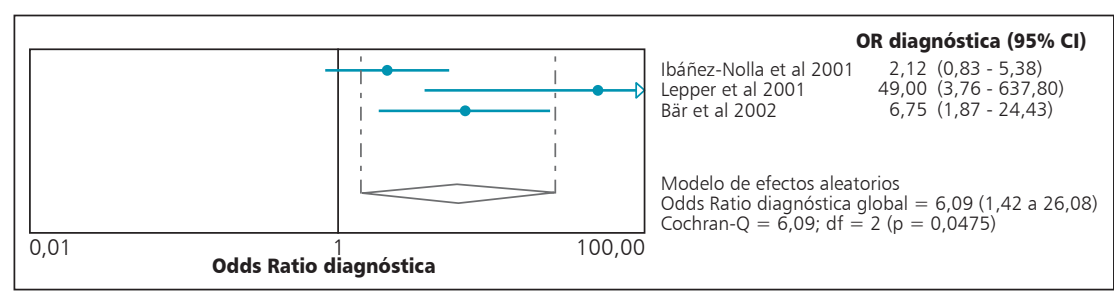

Figura 2. Odds Ratio diagnóstica para pruebas de detección de anticuerpos y diagnóstico de candidemia en pacientes en UCl.

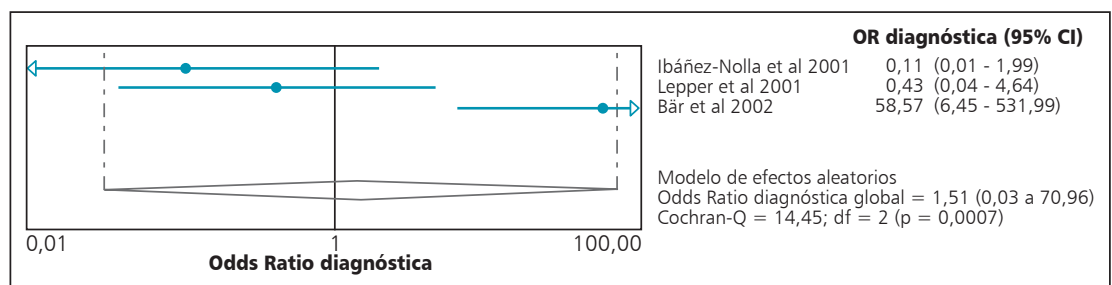

Figura 3. Odds Ratio diagnóstica para pruebas de detección de antígenos y diagnóstico de candidemia en pacientes en UCl.

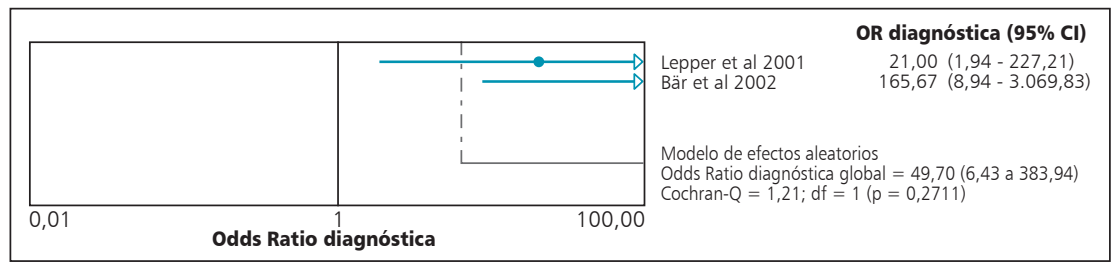

Figura 4. Odds Ratio diagnóstica para pruebas de detección de anticuerpos y antígenos en diagnóstico de candidemia en pacientes en $\mathrm{UCl}$.

\section{Discusión}

En esta revisión sistemática encontramos que las pruebas basadas en biología molecular tienen el mejor rendimiento diagnóstico, comparadas con las pruebas basadas en serología, pero no hay suficiente cuerpo científico que soporte ampliamente estos hallazgos en los pacientes hospitalizados en las UCI.

Las pruebas de biología molecular para el diagnóstico de candidemia en pacientes internados en UCI tienen un futuro promisorio por cuanto tienen alto rendimiento diagnóstico como lo evidencia el estudio de Mc Mullan y cols ${ }^{12}$; sin embargo, dicho rendimiento es óptimo cuando los predictores clínicos aplican a los pacientes en alto riesgo o con diagnóstico probado, pero pierde esta cualidad cuando se realiza en aquellos pacientes clasificados como probables, lo que se evidencia por la disminución de la sensibilidad de 87 a $72 \%$ cuando se evalúan los sujetos de estudio y no las muestras tomadas. Una desventaja que muestra la RPC-T R es que sólo se han construido los partidores (primers) de ciertas especies de Candida y se demostró que se presentan en los pacientes con candidiasis probada clínicamente algunas especies que no hibridaron y por tanto se descartan, pudiendo ser causantes del deterioro clínico.

Las pruebas diagnósticas basadas en medición de anticuerpos mostraron un pobre desempeño en el análisis global ya que tuvieron una baja sensibilidad aunque una alta especificidad que nos sugiere que estas pruebas podrían tener su mayor utilidad para mejorar la probabilidad diagnóstica de la enfermedad. La ORD para candidemia fue de 6 y estadísticamente significativa. Al revisar teóricamente esta prueba encontramos la dependencia de dichas pruebas del estado inmunológico del paciente, que en el paciente crítico puede estar seriamente comprometido.

Las pruebas de antigenemia igualmente se comportaron con baja sensibilidad; sin embargo, las mejorías en cuanto a significancia estadística fueron evidentes, la especificidad y los LR, tanto positivos como negativos, 
presentaron significancia estadística. Sin embargo, consideramos que la presencia de cambios en algunos estudios con respecto a la prueba utilizada así, como los puntos de corte para positividad y las diferencias en predicciones clínicas utilizadas pudieron afectar estos datos. Creemos que aún con el análisis de efecto umbral hecho con los pocos sujetos recolectados en el análisis global, dicho efecto de los puntos de corte afectó el rendimiento diagnóstico ya que esta prueba ha mostrado mejor resolución en otros estudios y en otros escenarios fuera de la UCI.

El análisis de las pruebas en conjunto que, como se explicó anteriormente, sólo se pudo realizar en dos estudios, nos mostró que la complementariedad de dichas pruebas sí mejoraron la sensibilidad, especificidad, LR+ y LR-, así como en la ORD, demostrando que la utilización de dichas pruebas en paralelo nos daría más certeza para inicios de tratamientos en los pacientes en riesgo, minimizando el uso de recursos innecesarios y evitando de esta manera riesgos adicionales en pacientes ya muy comprometidos como son los críticamente enfermos. Sin embargo, se presentó una pobre significancia estadística que puede mejorarse agregando al análisis estudios que aborden el diagnóstico serológico con más pacientes, mejor distribución aleatoria y nuevas pruebas desarrolladas. Creemos que estas pruebas pueden ser una alternativa diagnóstica eficiente y eficaz al diagnóstico molecular.

La posibilidad de llegar a un diagnóstico temprano en pacientes con factores de riesgo para candidemia ofrecería una oportunidad de inicio de tratamiento precoz ya que un reporte reciente, Patel y cols ${ }^{13}$, mostró que sólo aquellos pacientes que recibieron su tratamiento en las primeras 15 horas del diagnóstico mejoraron la sobrevida. Sin embargo, ninguno de los estudios analizados reportó mejor rendimiento en cuanto al diagnóstico temprano por laboratorio, lo que sería primordial para que el paciente en riesgo reciba tratamiento temprano. Sólo el estudio de McMullan y cols, basado en RPC-TR reportó el tiempo de diagnóstico comparado con candidemia probada entre -1 y +5 días.

Los costos en los que se incurre en el tratamiento de candidemia de manera empírica son elevados y llegan hasta el orden de más de 26 millones de dólares americanos al año por cuenta sólo de aplicar tratamiento antifúngico, estancia en UCI y mortalidad ${ }^{14}$. Por esto, consideramos que se hace necesario elaborar estudios con pruebas que tengan adecuado rendimiento, con las que se pueda llegar a un diagnóstico temprano, incluso en la etapa preclínica, lo cual evitaría en aquellos con factores de riesgo tratamientos inadecuados y retraso en los tratamientos que sean necesarios.

La presencia de predictores clínicos para pacientes en riesgo y que definen hoy en día, en ausencia de cultivos o demostración serológica de candidemia, la indicación de tratamiento empírico, no han mostrado que mejoren los resultados en cuanto a desenlaces primarios. Esto lo reporta Schuster y cols $^{15}$, en su artículo de 2008 donde encontraron que el inicio empírico de fluconazol comparado con placebo no mostró beneficio en dichos desenlaces (resolución de fiebre, ausencia de infección fúngica, ausencia de reacciones tóxicas, no cambio a otros antifúngicos sistémicos). Esto apoya nuestra conclusión sobre la necesidad de pruebas diagnósticas tempranas sensibles que nos permitan iniciar dichos tratamientos en quienes realmente se necesiten.

Se necesitan más estudios sobre pruebas diagnósticas para candidemia en pacientes críticos, en las diferentes metodologías de diagnóstico ya sean moleculares y serológicas, pero esos nuevos estudios deben enfocarse hacia mejorías en metodología, sujetos de estudio, pruebas de diagnóstico temprano y costo-efectividad de tratamiento empírico versus el guiado por laboratorio.

\section{Resumen}

La candidemia es una patología con alta morbilidad y mortalidad, especialmente en los pacientes sometidos a servicios de cuidado crítico. El diagnóstico precoz permite realizar tratamiento temprano. Objetivos: Realizar una revisión sistemática de la literatura para establecer cuáles son las pruebas de laboratorio con mejor rendimiento diagnóstico y operativo para el diagnóstico de candidemia en cuidado intensivo. Materiales y Métodos: Se realizó una revisión sistemática de la literatura disponible en PubMed, se sometieron a meta-análisis estudios de pruebas serológicas en MetaDisc-Beta 1.1.1. Resultados: Se incluyeron 4 estudios de 1.286 revisados, 3 de pruebas serológicas y 1 de RPC-RT. La sensibilidad y especificidad fue de 87 y $100 \%$ para RPC-RT, 47,5 y 82,6\% para pruebas de anticuerpos, 96 y $81 \%$ para pruebas de antígeno y anticuerpo. La ORD de antigenemia 1,51(IC95\% = 0,03270,964; $\mathrm{p}=0,001)$. Conclusiones: RPC-RT tiene mejor rendimiento diagnóstico, la medición de antigenemia más anticuerpos mejora la sensibilidad, especificidad, LR+ y LR-. No hay suficiente evidencia que soporte esto.

\section{Referencias}

1.- Pappas P G. Invasive candidiasis. Infect Dis Clin North Am 2006; 20: 485-506.

2.- Ostrosky-Zeichner L, Pappas P G. Invasive candidiasis in the intensive care unit. Crit Care
Med 2006; 34: 857-63.

3.- Zaoutis T E, Prasad P A, Localio A R, Coffin S E, Bell L M, Walsh T J, et al. Risk factors and predictors for candidemia in pediatric intensive care unit patients: implications for prevention. Clin Infect Dis 2010; 51: e38-45.
4.- Cortés J A, Reyes P, Gómez C, Buitrago G, Leal A L. Fungal bloodstream infections in tertiary care hospitals in Colombia. Rev Iberoam Micol 2011; 28: 74-8.

5.- Bouza E, Muñoz P. Epidemiology of candidemia in intensive care units. 
Int J Antimicrob Agents 2008; 32 Suppl 2: S87-91.

6.- Weinberger M, Leibovici L, Pérez S, Samra Z, Ostfeld I, Levi I, et al. Characteristics of candidaemia with Candida-albicans compared with non-albicans Candida species and predictors of mortality. J Hosp Infect 2005; 61: 146-54.

7.- $\quad$ Falagas M E, Apostolou K E, Pappas V D. Attributable mortality of candidemia: a systematic review of matched cohort and casecontrol studies. Eur J Clin Microbiol Infect Dis 2006; 25: 419-25.

8.- Liberati A, Altman D G, Tetzlaff J, Mulrow C, Gotzsche P C, Ioannidis J P, et al. The PRISMA statement for reporting systematic reviews and meta-analyses of studies that evaluate health care interventions: explanation and elaboration.
Ann Intern Med 2009; 151: W65-94.

9.- Ibáñez-Nolla J, Torres-Rodríguez J M, Nolla M, León M A, Méndez R, Soria G, et al. The utility of serology in diagnosing candidosis in non-neutropenic critically ill patients. Mycoses 2001; 44: 47-53.

10.- Lepper P M, Wiedeck H, Geldner G, Essig A, Trautmann M. Value of Candida antigen and antibody assays for the diagnosis of invasive candidosis in surgical intensive care patients. Intensive Care Med 2001; 27: 916-20.

11.- Bar W, Hecker H. Diagnosis of systemic Candida infections in patients of the intensive care unit. Significance of serum antigens and antibodies. Mycoses 2002; 45: 22-8.

12.- McMullan R, Metwally L, Coyle PV, Hedderwick S, McCloskey B, O’Neill H J, et al. A prospective clinical trial of a real- time polymerase chain reaction assay for the diagnosis of candidemia in nonneutropenic, critically ill adults. Clin Infect Dis 2008; 46: 890-6.

13.- Patel G P, Simon D, Scheetz M, Crank C W, Lodise T, Patel N. The effect of time to antifungal therapy on mortality in candidemia associated septic shock. Am J Ther 2009; 16: 508-11.

14.- Hassan I, Powell G, Sidhu M, Hart W M, Denning D W. Excess mortality, length of stay and cost attributable to candidaemia. J Infect 2009; 59: 360-5.

15.- Schuster M G, Edwards J E, Sobel J D, Darouiche R O, Karchmer A W, Hadley S, et al. Empirical fluconazole versus placebo for intensive care unit patients: a randomized trial. Ann Intern Med 2008; 149: 83-90. 